\title{
Complicated Inguinal Hernia in Children: An Experience in a Developing Country
}

\author{
Kevin E Chukwubuike
}

\begin{abstract}
Aim: To determine the demographic characteristics, clinical presentation, and management outcome of children treated for complicated inguinal hernia at a tertiary hospital in Enugu, Nigeria.

Materials and methods: This was a retrospective analysis of children who were managed for complicated inguinal hernia.

Results: Forty-eight cases of the complicated inguinal hernias had an emergency surgery during the study period. There were 34 males (70.8\%) and 14 females (29.2\%). The ages of the patients ranged from 1 month to 48 months, with a median age of 7.5 months. Sixty percent of the inguinal hernias were right sided and $40 \%$ were left sided. None was bilateral. The median time interval from the time of first clinic visit to incarceration was 6.5 months (range: 1-48). Twenty-one percent of the patients visited the clinic at least once before the incarceration. Pain over the inguinal swelling was present in all the patients. Surgical site infection (10.4\%) was the most common postoperative complication. Mortality was $2.1 \%$. Conclusion: Treatment of complicated inguinal hernia is associated with morbidity and mortality especially in developing countries due to late presentation.

Clinical significance: The importance of early treatment of inguinal hernia in children to avoid complications that may arise.

Keywords: Case series, Children, Complicated, Developing country, Hernia, Inguinal.

The Journal of Medical Sciences (2020): 10.5005/jp-journals-10045-00140
\end{abstract}

\section{INTRODUCTION}

Inguinal hernia manifests as a bulge in the groin and is usually noticed by the parents or pediatricians. Inguinal hernia is one of the most common clinical conditions seen by the pediatric surgeon. ${ }^{1}$ Incidence of inguinal hernia in the general population ranges from 0.8 to $5 \% .{ }^{2}$ In low-birth-weight and premature neonates, the incidence is reported to be up to $30 \% .^{3,4}$ Uncomplicated inguinal hernias are repaired as an elective surgical procedure with good results. Three to sixteen percent of pediatric inguinal hernias may develop complications in the form of incarceration, obstruction, and/or strangulation. ${ }^{5,6}$ The risk of inguinal hernia complications is more in infants because of the tighter inguinal ring that may trap the herniating bowel. Management of complicated inguinal hernia is associated with lots of morbidity such as bowel injury, injury to the vas deferens and testicular vessel, and surgical site infection. ${ }^{5,7}$ These complications are more common in developing countries due to delays in seeking medical care. ${ }^{8}$ The aim of this study was to determine the demographic characteristics, clinical presentation, and management outcome of children treated for complicated inguinal hernia at a tertiary hospital in Enugu, Nigeria.

\section{Materials and Methods}

This was a retrospective study of children aged 15 years and below who had surgery for complicated inguinal hernia between January 2008 and December 2017 at the pediatric surgery unit of Enugu State University Teaching Hospital (ESUTH) Enugu, Nigeria. For the purposes of this study, complicated inguinal is defined as an inguinal hernia that is incarcerated (irreducible), obstructed, or strangulated. Patients who underwent surgery for inguinal hernia at a peripheral hospital before referral to ESUTH for reoperation were excluded from this study. ESUTH is a tertiary hospital located in Enugu, South East Nigeria. The hospital serves
Department of Surgery, Pediatric Surgery Unit, Enugu State University Teaching Hospital, Enugu, Nigeria

Corresponding Author: Kevin E Chukwubuike, Department of Surgery, Pediatric Surgery Unit, Enugu State University Teaching Hospital, Enugu, Nigeria, Phone: +2348033834160, e-mail: chukwubuikeonline@yahoo.com

How to cite this article: Chukwubuike KE. Complicated Inguinal Hernia in Children: An Experience in a Developing Country. J Med Sci 2020;6(1):1-3.

Source of support: Nil

Conflict of interest: None

the whole of Enugu state, which according to the 2016 estimates of the National Population Commission and Nigerian National Bureau of Statistics, has a population of about 4 million people and a population density of $616.0 / \mathrm{km}^{2}$. The hospital also receives referrals from its neighboring states. Information was extracted from the case notes, operation notes, operation register, and admission-discharge records. The information extracted included age, gender, presenting symptom, duration of symptoms of complication before presentation to the hospital, duration of hernia prior to incarceration, intraoperative finding, operative procedure performed, complications of surgery, duration of hospital stay, and outcome of treatment. The protocol for managing incarcerated inguinal hernias in our center included administration of analgesics and sedatives, which aimed at achieving reduction of the hernia. Failure of hernia reduction or obvious signs of peritonitis are the indications for emergency surgery. The surgical approach for complicated inguinal hernia was through an inguinal incision, which was extended laterally to gain access to the peritoneal cavity (laparotomy) if the content of the hernia was not viable, for 
a resection. The hernia procedure performed was high ligation of the patent processus (herniotomy) with or without narrowing of the widened inguinal ring (herniorrhaphy).

The follow-up period was 12 months. Ethical approval was waived by the ethics and research committee of ESUTH due to retrospective nature of the study. The Statistical Package for the Social Science (SPSS) version 2, manufactured by IBM Corporation Chicago, Illinois, was used for data entry and analysis. Data were expressed as percentages, median, mean, and range.

\section{Results}

\section{Patients' Demographics}

A total of 1,477 cases of inguinal hernias were seen during the period of the study, out of which 192 cases (13\%) were irreducible. A total of 48 (25\%) cases, out of the 192, had an emergency inguinal hernia surgery and formed the basis of this report. The rest of the irreducible cases (75\%) had elective inguinal herniotomy following reduction of the inguinal hernias on administration of analgesics and sedatives. There were 46 males (95.8\%) and 2 females (4.2\%), which corresponds to a male to female ratio of 23:1. The ages of the patients ranged from 1 month to 48 months, with a median age of 7.5 months. Seventy-five percent of the patients were less than 12 months of age. Sixty percent of the inguinal hernias were right sided and $40 \%$ were left sided. None was bilateral. The median time interval from the time of first clinic visit to incarceration was 6.5 months (range: 1-48). Twenty-one percent of the patients visited the clinic at least once before the incarceration while $79 \%$ never visited the clinic and presented for the first time with symptoms of incarceration. On incarceration, the median duration of symptoms prior to presentation to the hospital was 2 days (range: $1-5$ ). All the patients who underwent surgery were operated upon within 24 hours of presentation to the hospital. The mean duration of hospital stay was 8 days (range: 4-11).

\section{Presenting Symptoms}

All the patients presented with pain over the inguinal swelling. The other symptoms included vomiting in 32 patients (66.6\%), constipation in 8 patients (16.7\%), and abdominal distension in 8 patients (16.7\%).

\section{Intraoperative Findings}

The bowel content of the hernia sac was found to be viable in 42 patients (87.5\%), whereas 6 patients $(12.5 \%)$ had nonviable bowel. Two patients (4.2\%) had gangrenous right testis resulting from pressure of the incarcerated bowel.

\section{Operative Procedure Performed}

The procedures performed in the patients are depicted in Table 1.

\section{Postoperative Complications}

Thirty-eight patients (79.1\%) did not develop any complications. The postoperative complications are shown in Table 2.

Table 1: Operative procedure performed

\begin{tabular}{lcc}
\hline Operation performed & Number & Percentage \\
\hline Reduction of viable bowel & 42 & 87.5 \\
Resection with end-to-end anastomosis & 6 & 12.5 \\
Orchidectomy & 2 & 4.2 \\
\hline
\end{tabular}

\section{Outcome}

Forty-four patients (91.6\%) did well and were discharged home. Three patients $(6.3 \%)$ signed out against medical advice in the postoperative period. Mortality was recorded in one patient (2.1\%), a neonate, due to aspiration pneumonitis. There was no recurrence.

\section{Discussion}

Inguinal hernias are the most common incarcerated hernias in children. ${ }^{9}$ Although unilateral inguinal hernias are more common in males than females, there is a higher incidence of bilateral inguinal hernias in females. ${ }^{5,6}$ With regards to the rate of incarceration of inguinal hernia, there is no difference between males and females. ${ }^{2,10}$ Complicated inguinal hernia is a surgical emergency that starts with incarceration of the herniating abdominal viscera with or without features of intestinal obstruction. This may progress to strangulation if the incarceration is not promptly relieved.

In the current study, the complicated inguinal hernia rate of $13 \%$ is in agreement with the finding of a similar study done in Ilorin, North Central Nigeria. ${ }^{11}$ However, Ameh reported a complication rate of $4.8 \%$ while Stylianos reported $9.4 \% ., 12$ Other studies have reported complication rates of between 12 and $16 \%{ }^{10,13}$ The reason for the differences in complication rates may be due to differences in the time of presentation to the hospital as reported by Ameh. ${ }^{12}$ The male dominance recorded in the present study is consistent with the report of other series on complicated inguinal hernia., 80,11 This male dominance may be explained by the higher incidence of unilateral inguinal hernias in males. ${ }^{9}$ Alrzoq recorded that male gender is a risk factor for inguinal hernia incarceration. ${ }^{14}$ There are wide variations in the median ages of children who presented with complicated inguinal hernia. The finding of 7.5 months in the current study is the average of the findings of Lawal et al. and Bamigbola et al. 8,11 However, a study conducted in Zaria, Northern Nigeria, reported that $80 \%$ of complicated inguinal hernias occurred in neonates. ${ }^{15}$ Stylianos reported that majority of their patients were less than 1 year, similar to our finding. ${ }^{5}$ The anatomy of the inguinal canal in infants may explain the increased incidence of complicated inguinal hernia in infants and younger children: Their deep and external inguinal rings are superimposed. This may explain the common occurrence of neonatal inguinal hernia incarceration. Complicated right inguinal hernia is more common than complicated left inguinal hernia. This finding was consistently observed in the current study and in other studies too. $8,11,14$ The left testis descends before the right testis and the left processus vaginalis (a diverticulum of the peritoneal membrane) closes before the right processus vaginalis. ${ }^{1,16}$ This may explain the higher incidence of right inguinal hernia. Thus, the higher incidence of complicated inguinal hernia on the right side. Twenty-one percent of the patients in the current study were scheduled for surgery before the complication occurred. This is comparable to the findings of Stylianos et al. and Bamigbola et

Table 2: Postoperative complications

\begin{tabular}{lll}
\hline Complications & Number & Percentage \\
\hline Surgical site infection & 5 & 10.4 \\
Recurrence of inguinal hernia & 3 & 6.3 \\
Wound dehiscence & 2 & 4.2 \\
\hline
\end{tabular}


al. ${ }^{5,11}$ The high volume of patients/ratio in developing countries may explain the challenge of operating on all inguinal hernias before complications arise. Late presentation of our patients when inguinal hernia complication occurs is evidenced by the 2-day lag period before presentation to the hospital. Bamigbola reported an 18-hour interval before presentation of the patients to the hospital. ${ }^{11}$ This delayed presentation may be due to poverty and ignorance that is prevalent in developing countries. The duration of hospital stay of our patients is longer than other published series. ${ }^{5,15}$ The differences in duration of hospital stay may be explained by the intraoperative finding and the surgical procedure performed.

The clinical symptoms of complicated inguinal hernia depend on the part of the bowel incarcerated in the hernia sac. Proximal bowel obstruction gives early vomiting and minimal abdominal distension while distal bowel obstruction gives more abdominal distension. ${ }^{17}$ The symptoms of pain over the inguinal mass, vomiting, abdominal distension, and constipation, which we recorded in the current study are similar to the reports of Bamigbola et al. ${ }^{11}$ However, if the incarceration progresses to strangulation, the child may have passage of bloody stool and hemodynamic instability. ${ }^{9}$

In the present study, the intestinal resection rate (for gangrenous bowel) is similar to the report of a study done in Zaria, Nigeria. ${ }^{12}$ However, intestinal resection rates as low as $6.2 \%$ and as high as $36 \%$ have been reported. ${ }^{12,18}$ The differences in the intestinal resection rate may be explained by the age differences of the patients recruited into the different studies. For instance, in two separate studies done in the same area, Ameh reported intestinal resection rate of $36 \%$ in neonates whereas the resection rate was $11 \%$ in children less than 2 years old. ${ }^{12,15}$ The orchidectomy rate, for testicular gangrene in the current study, of $4.2 \%$ is low when compared to other published series. ${ }^{11,12,15,18}$ It is worthy to note that in females the ovaries can be part of the wall of the hernia sac in sliding hernia. Turkyilmaz et al. reported $1.8 \%$ oophorectomy rate in their series. ${ }^{18}$

The complication rate we recorded is consistent with the reports of other studies with surgical site infection being the most common. ${ }^{11,12}$ Wound infection rates are higher in neonates. ${ }^{15}$ Other documented complications that may occur following surgery for incarcerated inguinal hernia include transection of the vas deferens, iatrogenic undescended testis, testicular atrophy, and recurrence. ${ }^{7}$ The mortality rate recorded in the present study tallies with the reports of other studies. ${ }^{11,18}$ However, Ameh reported a mortality of $5.9 \%$ in his series on inguinal hernias in neonates. ${ }^{15}$ Other studies recorded no mortalities. ${ }^{10,12}$ The reason for these variations in mortality rate is unknown but may be explained by the late presentation of the patient to the hospital. Bamigbola et al. reported that a patient may present with seizures due to electrolyte derangements following prolonged vomiting from complicated inguinal hernia. ${ }^{11}$

\section{Conclusion}

Majority of inguinal hernias in children are uncomplicated and repair of uncomplicated inguinal hernia is simple and straightforward. However, treatment of complicated inguinal hernia is associated with morbidity and mortality especially in developing countries due to late presentation. Improved awareness on the part of the parents and general practitioners will avoid delayed presentation.

\section{Limitations of this Study}

This study was limited by the small number of cases. A larger number of cases would have availed better analysis.

This study was a single institution experience, which may not be generalizable to other institutions and other countries.

\section{Clinical Significance}

The importance of early treatment of inguinal hernia in children to avoid complications that may arise.

\section{References}

1. Brandt ML. Pediatric hernias. Surg Clin North Am 2008;88(1):27-43. DOI: 10.1016/j.suc.2007.11.006.

2. Chang S-J, Chen JY-C, Hsu C-K, et al. The incidence of inguinal hernia and associated risk factors of incarceration in pediatric inguinal hernia: a nation-wide longitudinal population-based study. Hernia 2016;20(4):559-563. DOI: 10.1007/s10029-015-1450-x.

3. Burgmeier C, Dreyhaupt J, Schier F. Comparison of inguinal hernia and asymptomatic patent processus vaginalis in term and preterm infants. J Pediatr Surg 2014;49(9):1416-1418. DOI: 10.1016/j. jpedsurg.2014.03.013.

4. Lautz TB, Raval MV, Reynolds M. Does timing matter? A national perspective on the risk of incarceration in premature neonates with inguinal hernia. J Pediatr 2011;158(4):573-577. DOI: 10.1016/j. jpeds.2010.09.047.

5. Stylianos S, Jacir NN, Harris BH. Incarceration of inguinal hernia in infants prior to elective repair. J Peiatr Surg 1993;28(4):582-583. DOI: 10.1016/0022-3468(93)90665-8.

6. Rajput A, Gauderer MWL, Hack M. Inguinal hernias in very low birth weight infants: incidence and timing of repair. J Pediatr Surg 1992;27(10):1322-1324. DOI: 10.1016/0022-3468(92)90287-H.

7. Nah S, Giacomello L, Eaton S, et al. Surgical repair of incarcerated inguinal hernia: laparoscopic or open? Eur J Pediatr Surg 2011;21(01):811. DOI: 10.1055/s-0030-1262793.

8. Lawal TA, Egbuchulem KI, Ajao AE. Obstructed inguinal hernia in children: case-controlled approach to evaluate the influence of socio-demographic variables. J West Afr Coll Surg 2014;4(2):76-85.

9. Abdulhai SA, Glenn IC, Ponsky TA. Incarcerated pediatric hernias. Surg Clin N Am 2017;97(1):129-145. DOI: 10.1016/j.suc.2016.08.010.

10. Ein $\mathrm{SH}, \mathrm{Njere}$ I, Ein A. Six thousand three hundred sixty-one pediatric inguinal hernia: a 35-year review. J Pediatr Surg 2006;41(5):980-986. DOI: 10.1016/j.jpedsurg.2006.01.020.

11. Bamigbola KT, Nasir AA, Abdul-Rahman IO, et al. Complicated childhood inguinal hernias in UITH. Ilorin Afr J Surg 2012;9(3):227-230. DOI: 10.4103/0189-6725.104725.

12. Ameh EA. Incarcerated and strangulated inguinal hernias in children in Zaria, Nigeria. East Afr Med J 1999;76(9):499-501.

13. Gahukamble DB, Khamage AS. Early versus delayed repair of reduced incarcerated inguinal hernias in the pediatric population. J Pediatr Surg 1996;31(9):1218-1220. DOI: 10.1016/S0022-3468(96)90235-3.

14. Alrzoq RA, Mustafa AA, Alolayt IYI, et al. The incidence of inguinal hernia among children. Egypt J Hospital Med 2018;70(3):483-486.

15. Ameh EA. Morbidity and mortality of inguinal hernia in the newborn. Niger Postgrad Med J 2002;9(4):233-234.

16. Fraser JD, Synder CL. Inguinal hernias and hydroceles Ashcraft KW, Holcomb GW, Murphy JP, et al., ed. Ashcraft's pediatric surgery. 6th ed., London: Saunders/Elsevier; 2014. pp. 679-686.

17. Chukwubuike KE, Nduagubam OC, Eze TC. Adhesive intestinal obstruction in children: A 10 -year experience in a teaching hospital in Enugu. Nigeria Int J Anaesth Clin Med 2019;7(2):50-54. DOI: 10.11648/j. ijacm.20190702.15.

18. Turkyilmaz Z, Sonmez K, Karabulut R, et al. Incarcerated inguinal hernia in children. Hong Kong J Emerg Med 2010;17:244-249. 